\title{
RISK AVERSION BEHAVIOR. RELATIONSHIPS BETWEEN RISK AVERSION, PRUDENCE AND CAUTIOUSNESS
}

\author{
Professor PhD Pirtea Marilen, West University de Vest of Timi oara,marilen.pirtea@feaa.uvt.ro \\ Ec. Boțoc Claudiu, West University de Vest of Timi oara, claudiu.botoc@feaa.uvt.ro
}

\begin{abstract}
This paper defines decreasing absolute risk aversion in purely behavioral terms without any assumption of differentiability and shows that a strictly increasing and risk averse utility function with decreasing absolute risk aversion is necessarily differentiable with an absolutely continuous derivative. A risk averse utility function has decreasing absolute risk aversion if and only if it has a decreasing absolute risk aversion density, and if and only if the cumulative absolute risk aversion function is increasing and concave. This leads to a characterization of all such utility functions. Analogues of these results also hold for increasing absolute and for increasing and decreasing relative risk aversion.
\end{abstract}

Keywords: risk aversion, prudence, cautiousness

JEL codes: $G 32$

\section{Introduction}

Decreasing absolute risk aversion means that the decision maker behaves in a less risk averse fashion the larger his wealth. Pratt [3] defined it by the condition that the equivalent risk premium $\pi(\mathrm{x}, \mathrm{z})$ should be a decreasing function of initial wealth $\mathrm{x}$, for every random addition $\mathrm{z}$ to wealth. Dybvig and Lippman [2], following Yaari [7], defined decreasing risk aversion by requiring that "gambles accepted at a given level of wealth will be accepted at all higher levels of wealth." These definitions are equivalent, and they are behavioral in the sense that they can be directly interpreted in terms of preferences or choice behavior. An important fact about decreasing absolute risk aversion is that it can be expressed in terms of the Arrow-Pratt coefficient of absolute risk aversion.

Indeed, a utility function exhibits decreasing absolute risk aversion if and only if the coefficient of absolute risk aversion is decreasing. This was shown by Pratt [3] and by Dybvig and Lippman [2]. Arrow [1] stated this result as well, except that his terminology was inverted relative to Pratt's. Arrow used the decreasing coefficient of absolute risk aversion as a definition of decreasing absolute risk aversion, and he then argued that it is equivalent to more risk averse behavior at higher wealth levels.

The definition of the coefficient of absolute risk aversion assumes that the utility function is twice differentiable. Pratt's proof that one utility function is more risk averse than another if and only if it globally has a larger coefficient of risk aversion assumes that the utility functions are twice continuously differentiable. The same assumption therefore underlies the proof that decreasing absolute risk aversion is equivalent to a decreasing coefficient of absolute risk aversion. Since Dybvig and Lippman use Pratt's results, their proof also relies on this assumption.

However, a utility function with decreasing absolute risk aversion is not necessarily twice continuously differentiable If it is strictly increasing, then it has to be everywhere differentiable with positive derivative. If, in addition, it is concave and differentiable, then it is automatically continuously differentiable; but the second derivative may not exist everywhere. This paper defines 
the concept of an absolute risk aversion density, which is a generalization of the coefficient of absolute risk aversion. The paper's main result, Theorem 1, says that a strictly increasing risk averse utility function has decreasing absolute risk aversion if and only if it has a decreasing absolute risk aversion density. It generalizes the result of Pratt [3], Arrow [1], and Dybvig and Lippman [2] to utility functions that are not assumed to be twice continuously differentiable.

Specifically, if the utility function is strictly increasing and risk averse, and if it exhibits decreasing absolute risk aversion, then the marginal utility function is absolutely continuous, which means that it has a density function. Since the marginal utility function is absolutely continuous, the same is true of what we call the cumulative absolute risk aversion function, the negative of the logarithm of the marginal utility. This allows us to define the absolute risk aversion density as the density of the cumulative absolute risk aversion function. It equals the negative of the density of the marginal utility divided by the marginal utility itself, and it coincides with the usual coefficient of absolute risk aversion whenever the latter is defined. It is unique almost everywhere.

An idea similar to the absolute risk aversion density appeared in Vickson [4], [5], [6], where a DARA utility function was defined as one which has, in our terminology, a non-negative, decreasing, and "piecewise smooth" absolute risk aversion density.

Theorem 1 also says that a strictly increasing risk averse utility function has decreasing absolute risk aversion if and only if its cumulative absolute risk aversion function is increasing and concave. This allows a complete characterization of strictly increasing risk averse utility functions with decreasing absolute risk aversion. The characterization says that such a utility function is uniquely determined by its cumulative absolute risk aversion function, which can be any increasing concave function, and an additive constant. This is so because the utility function can be recovered, up to an additive constant, by integrating the exponential of the cumulative absolute risk aversion function.

Pratt [3] wrote that ". . . , convenient utility functions for which [the coefficient of absolute risk aversion] is decreasing are not so very easy to find." Our characterization represents a way of finding all such utility functions.

All the results hold for increasing absolute risk aversion as well, except that the cumulative absolute risk aversion function will be convex rather than concave, and the absolute risk aversion density will be increasing rather than decreasing. Thus, a strictly increasing risk averse utility function has increasing absolute risk aversion if and only if its cumulative absolute risk aversion function is increasing and convex, and if and only if it has an increasing absolute risk aversion density.

Analogues of all the results also hold for relative risk aversion, where increasing or decreasing relative risk aversion is defined in behavioral terms without assuming that the utility function is twice differentiable. The cumulative relative risk aversion function of a utility function defined on the positive half-line is defined as the composition of the exponential function and the cumulative absolute risk aversion function. If it is absolutely continuous, then we call its density the relative risk aversion density.

If the utility function is strictly increasing and exhibits increasing or decreasing relative risk aversion, then it is necessarily differentiable with positive derivative, so that the cumulative absolute and the cumulative relative risk aversion functions are well defined. If, in addition, the utility function is risk averse, then the marginal utility functions, the cumulative absolute and the cumulative relative risk aversion functions are all absolutely continuous.

Theorem 2 says that a strictly increasing risk averse utility function has increasing (decreasing) relative risk aversion if and only if it has an increasing (decreasing) relative risk aversion density. The theorem also says that a strictly increasing risk averse utility function has increasing (decreasing) relative risk aversion if and only if its cumulative relative risk aversion function is increasing and convex (concave). This implies that a strictly increasing risk averse utility function with increasing (decreasing) absolute risk aversion is uniquely determined by its 
cumulative absolute risk aversion function, which can be any increasing and convex (concave) function, and an additive constant.

\section{Some Concepts}

The concepts risk aversion, prudence and cautiousness have interpretations for investor's main activities in financial markets, namely investment, saving, and hedging respectively. The relationships between these concepts reveals that a more absolutely prudent investor is almost naturally more absolutely risk averse and (along his optimal risksharing rule) a more cautious investor will be less relative risk averse. Parallel to Kimball's finding that decreasing absolute prudence implies decreasing absolute risk aversion, it is found that increasing (decreasing) cautiousness implies decreasing (increasing) relative risk aversion.

\subsection{Risk Aversion}

Absolute risk aversion: As interpreted by Pratt (1964), the function

$$
R(x)=-\frac{u^{\prime \prime}(x)}{u^{\prime}(x)}
$$

measures the strength of an investor's motives to demand risk premium for a small and actuarially neutral risk. Precisely, the risk premium demanded by an investor with utility $\mathrm{u}(\mathrm{x})$ will be the function $\mathrm{R}(\mathrm{x})$ times half the variance of the risk. It was also shown to be a global measure of risk aversion in the sense that if the function $\mathrm{R}(\mathrm{x})$ of an investor is always larger than that of the other, then the former will demand a larger risk premium than the later for any risk at any wealth level.

Decreasing Absolute Risk Aversion: It has been agreed widely in the literature that an investor.s utility is most likely to disclose decreasing absolute risk aversion or DARA. DARA means equivalently that an investor's risk premium is a decreasing function of his wealth for any certain level of risk. It has been shown by Kihlstrome, Romer and Williams (1981) that DARA ensures the validity of the risk aversion measurement in the case of random initial wealth.

Relative risk aversion: As also interpreted by Pratt (1964), the function

$$
\gamma(x)=x \times R(x)
$$

measures strength of an investor's motives to demand the risk premium proportional to his wealth for a small and actuarially neutral risk. The more relative risk averse an investor is, the more risk premium proportional to his wealth he will demand. The concept of relative risk aversion draws more attention after the establishment of the discrete time Black-Scholes option pricing model based on the assumption of constant relative risk aversion.

\subsection{Prudence}

Absolute prudence: Kimball (1990) introduced a theory of precautionary saving closely analogous to the theory of risk aversion based on the concept of prudence. Absolute prudence is defined as:

$$
P(x)=-\frac{u^{\prime \prime}(x)}{u^{\prime \prime}(x)}
$$


It measures the strength of an investor's motives to demand equivalent precautionary premium for a small and actuarially neutral risk. The more absolute prudent an investor is, the more equivalent precautionary premium he will demand for a certain level of risk.

Decreasing Absolute Prudence: DAP or decreasing absolute prudence means equivalently that an investor's equivalent precautionary premium is a decreasing function of his wealth for any certain level of risk. If utility $\mathrm{u}(\mathrm{x})$ has decreasing absolute prudence, then it is equivalent to that $-\mathrm{u}^{\prime}(\mathrm{x})$ has decreasing absolute risk aversion.

Standard risk aversion: Kimball (1993) introduced the concept of standard risk aversion. A von Neumann-Morgenstern utility function has standard risk aversion if every risk that has a negative interaction with a small reduction in wealth also has a negative interaction with any undesirable, independent risk. It was shown that the combination of DAP and DARA is the necessary and sufficient condition for standard risk aversion.

\subsection{Cautiousness}

Cautiousness: As interpreted by Huang (1999), cautiousness defined as:

$$
C(x)=\frac{P(x)}{R(x)}
$$

is a measure of the strength of an investor's motives to hedge the downside risk of his investment using convex-payoff contracts. It was shown in a HARA economy that the more cautious an investor is, the eager he is to hedge with convex-payoff contracts. Given an increasing and concave utility function $\mathrm{u}(\mathrm{x})$, we have that:

$$
\left(\ln R(x)^{\prime}=\left(\ln -u^{\prime \prime}(x)^{\prime}-\left(\ln u^{\prime}(x)^{\prime}=-(P(x)-R(x))\right.\right.\right.
$$

It follows that:

$$
\left(\frac{1}{R(x)}\right)^{\prime}=-\frac{R^{\prime}(x)}{R^{2}(x)}=C(x)-1
$$

Thus cautiousness can be alternatively defined as:

$$
\left(\frac{1}{R(x)}\right)^{\prime}+1
$$

Simple calculation also reveals that DARA is equivalent to $\mathrm{C}(\mathrm{x})>1$ since $\mathrm{R}^{\prime}(\mathrm{x})<0$ is equivalent to $\mathrm{P}(\mathrm{x})>\mathrm{R}(\mathrm{x})$.

Increasing (decreasing) cautiousness: Increasing (decreasing) cautiousness can be interpreted as an investor's eagerness to hedge the downside risk of his investment with convexpayoff contracts is increasing (decreasing) in his wealth. Intuitively, it seems more reasonable for an investor to have decreasing cautiousness since when he becomes richer, he might be less eager to buy puts on the bad states to hedge the downside risk. However, until now we do not have empirical evidence about this conclusion. For a utility with positive first three order derivatives, we have that

$$
(\ln C(x))^{\prime}=(\ln P(x))^{\prime}-(\ln R(x))^{\prime} .
$$

Since: 


$$
(\ln R(x))^{\prime}=-(P(x)-R(x))
$$

and :

$$
(\ln P(x))^{\prime}=-(T(x)-P(x))
$$

where $\mathrm{T}(\mathrm{x})$ is temperance defined as $T(x)=-\frac{u^{\prime \prime \prime \prime}(x)}{u^{\prime \prime \prime}(x)}$, it follows that:

$$
(\ln C(x))^{\prime}=-(T(x)-P(x))+(P(x)-R(x))
$$

Thus increasing (decreasing) cautiousness is equivalent to:

$$
\mathrm{T}(\mathrm{x})-\mathrm{P}(\mathrm{x})<\mathrm{P}(\mathrm{x})-\mathrm{R}(\mathrm{x})
$$

An investor's decisions on precautionary saving and investment in risky assets must be closely related. If it is desirable for an investor to make more precautionary saving than the other facing the same level of uncertainty in the same future income, then it is, perhaps, naturally undesirable for the former to invest more in the risky assets than the later, since this will lead to higher level of uncertainty in the future income of the former than that of the later. In the early days, the difference between investor's motivation of precautionary saving and risk aversion was sometimes even ignored. As noted by Leland (1968), "when rigor is absent, economists have tended to equate the precautionary demand for saving with the concept of risk avoidance". Thus the development of the concept of prudence by Kimball (1990) makes a significant contribution to the theory of precautionary saving. Yet it is interesting to see that the two concepts, absolute prudence and absolute risk aversion, have a close relationship between each other.

\section{Recent evolution on Romanian capital market}

2007 was a good year for investors to the stock market. With an increase of the BET index of $22.1 \%$ shares on the exchange brought yields average three times higher than bank interest rates. BET index performance in 2007 is very similar to that recorded in 2006, when the index rose by $22.2 \%$. Like in 2006, and this year the shares listed in category II of BVB have been performing: BET-C increased in 2007 to $32.6 \%$ (28.5\% in the previous year). If actions listed on the stock market have brought, in general, performing similar to those of 2006, the major difference in performance appears on Rasdaq, after a price appreciation continue to BSE in recent years, investors have discovered many shares relatively cheap but with potential for Rasdaq, inducing a Growth index compozit market, Rasdaq-C of 96.5\% - the highest performance of the index from its launch.

Transaction values the shares listed on the BVB increased by $40 \%$ in 2007 to 13.8 billion, the value of transactions Rasdaq shares rose even more spectacular: 4.3 billion - an increase of more than five times the face value of 2006 . Through these performance in 2007 can be considered the market Rasdaq: even if the value of transactions with shares Rasdaq remains only $31 \%$ of the transactions with shares BVB, and capitalization is $28 \%$ of the BVB, the jump of vision Rasdaq of issuers has been remarkable this year.

Achievements capital markets in 2007 include the successful conclusion of the largest initial public offerings in history, the SNTGN Transgaz, which attracted total amount of 1.8 billion euros (issue has recorded a premiere: trading rights allocation ) Successfully conduct the local market a 
prime bonds of the European Investment Bank, the second financial institution that attract international funds on Romanian capital market after the World Bank; launch gold futures contract on BMFMS and the platform of derivatives trading at BVB.

But the situation started this to get worse in the second half of 2007 when the BET of the most important 10 companies on stock exchange lost $48 \%$ against the maximum reached on 24 July 2007, while SIF sites recorded a decline of average $60 \%$ compared to the same level of reference, as seen in figure no 1 :

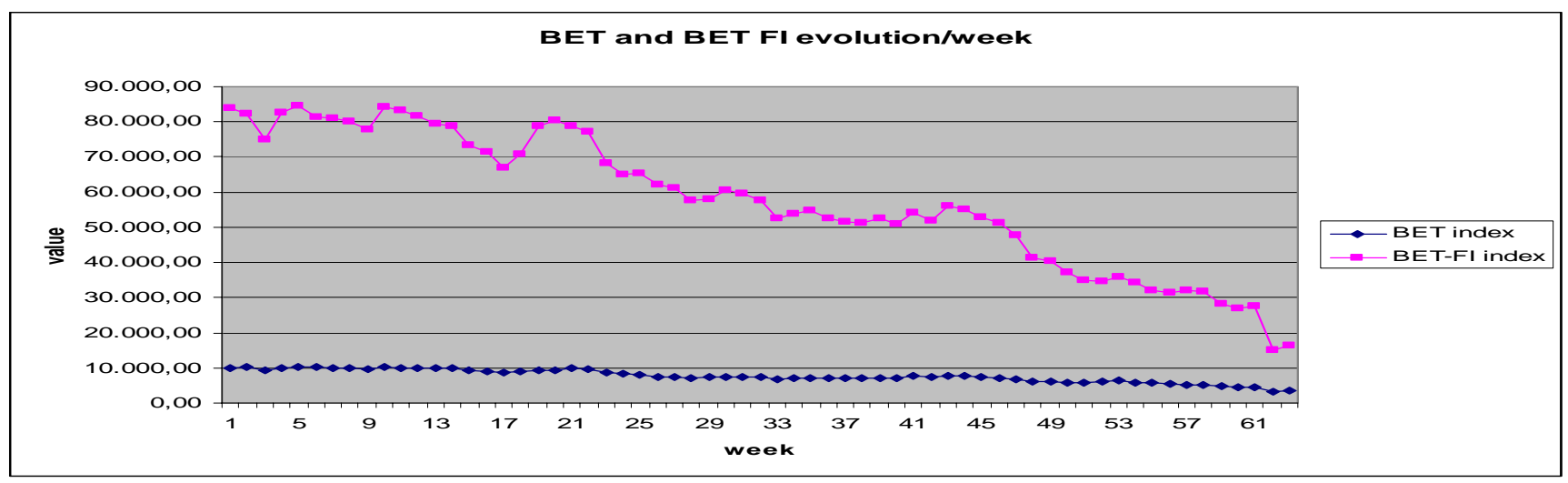

Fig. no 1 - BET and BET FI evolution on BVB during august 2007-october 2008

Source: $\underline{w w w . b v b . r o}$

About 30 companies on the stock exchange lost in the last year more than half of the market. Of these, most are from the financial sector, namely the five SIF's and Broker Cluj. No sector has not escaped, but the falls, pharmaceutical companies and Biofarm Zentivaa, oil company Rompetrol Rafinarie, the carrier Transelectrica energy, as well as equipment manufacturers and Turbomecanica and Compa losing over 50\% of highest level in July 2007. However, the market falls dramatically from day to day, and indices establish new minimum. Brokers show the finger to local investors who have lost patience and now without selling to take consider how prices are or how big are losses, as seen in Figure no 2 and no 3:

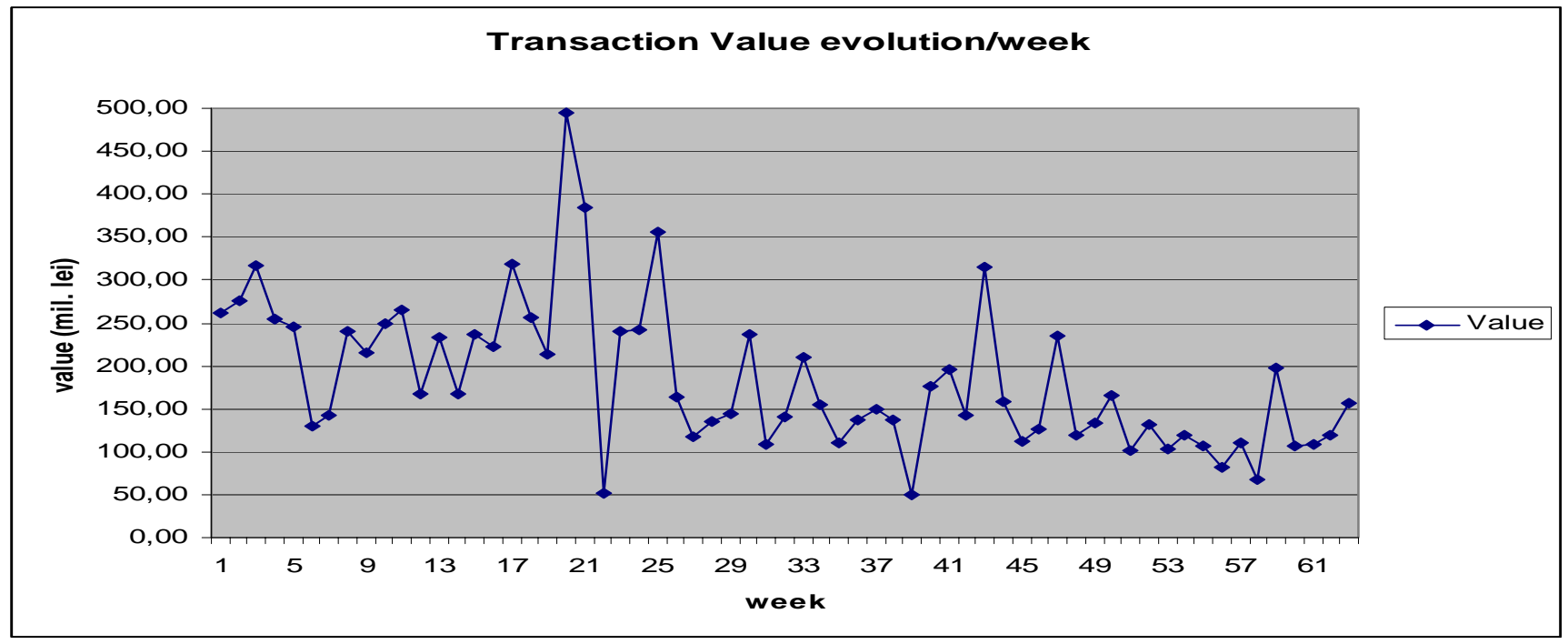

Fig. no 2 - Transaction values evolution on BVB during august 2007-october 2008

Source: $\underline{w w w . b v b . r o}$ 


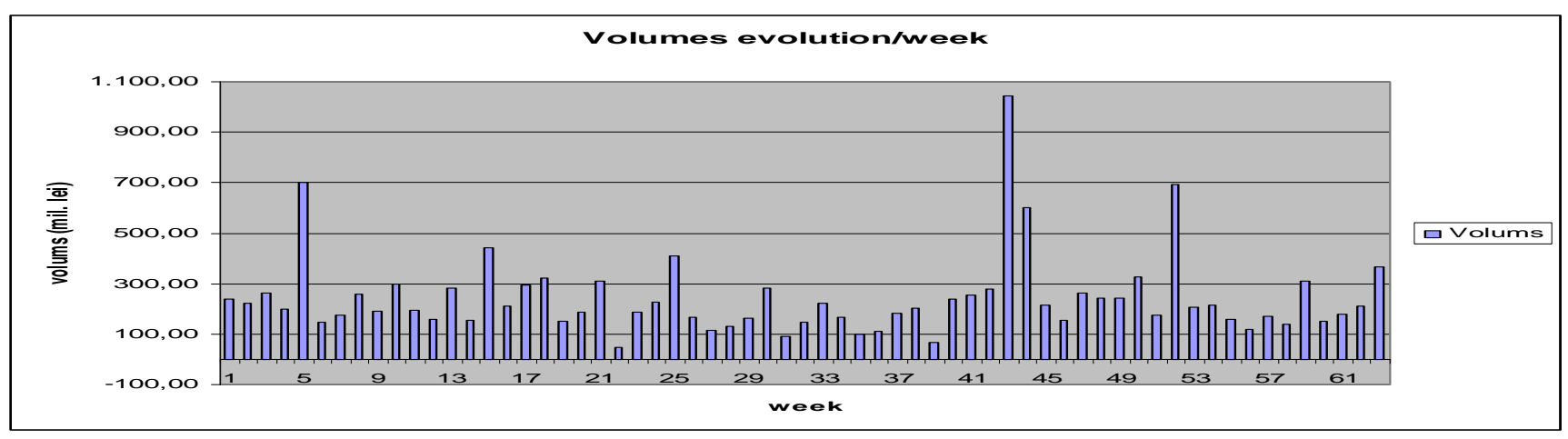

Fig. no 3 - Transactions volumes evolution on BVB during august 2007-october 2008 Source: $\underline{w w w . b v b . r o}$

In the absence of foreign funds, which do not follow the Romanian market, and local institutional investors, who now almost there, sales of small investors find it hard counterparty, where increase dramatically. Lack of institutional investors on the market could prolong the crisis on the Stock Exchange in coming months, in conditions that few investors still dare to buy now, knowing he could lose 5-6\% in one day. Even investors long-term think several times before buying despite attractive prices on some issuers.

During this period, so fundamental analysis and the technique has a role increasingly small in making decisions, investors acting more on the court always feeling. Scare canceled long-term and sell at any price. If you look historically at least, all those who in the past have been up on any market they had to win betting on a return of stock exchange.

One factor that could lead to a return would be a veering large investors about emerging markets, namely, a reconsideration of the importance of these markets. In addition, the levels at which we got right now are very low compared with the economic performance of the issuers. Brokers no longer throw but his prognosis might come when the long awaited return of the stock exchange, the external situation, in their view, the determining factor for the market evolution local capital

\section{Conclusion}

This paper derives close relationships between risk aversion, prudence and cautiousness. It is shown that a more absolute prudent investor is almost naturally more absolute risk averse and (along the optimal risk-sharing rule) a more cautious investor will be less relative risk-averse. Parallel to Kimball's Finding that DAP implies DARA, it is found that increasing cautiousness implies decreasing relative risk aversion.

Since prudence defined as the function $P(x)=-\frac{u^{\prime \prime \prime}(x)}{u^{\prime \prime}(x)}$ and cautiousness defined as the function: $C(x)=\frac{P(x)}{R(x)}$ involve higher order derivative of the utility function $\mathrm{u}(\mathrm{x})$ than risk aversion defined as the function $R(x)=-\frac{u^{\prime \prime}(x)}{u^{\prime}(x)}$, it is not surprising that both prudence and cautiousness have implications for risk aversion. Mathematically, the higher order derivatives should have implications for the lower order derivatives given certain boundary conditions. However, with the same reason it is very difficult to find the significant implications of risk aversion for prudence and cautiousness.

Further work will be interesting if we can show the implication of risk aversion for prudence and the implication of relative risk aversion for cautiousness imposing certain conditions. 


\section{References:}

1. Arrow, K. J., Aspects of a theory of risk bearing. Yrjo Jahnson Lectures, Helsinki, 1965. Reprinted in $[2,1971]$.

2. Dybvig, P. H., Lippman. S. A. ,An alternative characterization of decreasing absolute risk aversion. Econometrica, , 1983.

3. Pratt, J. W., Risk aversion in the small and in the large, Econometrica, 1964.

4. Vickson, R. G. , Stochastic dominance for decreasing absolute risk aversion, Journal of Financial and Quantitative Analysis, 1975.

5. Vickson., R. G., Stochastic dominance tests for decreasing absolute risk aversion I: Discrete random variables, Management Science, August 1975.

6. Vickson., R. G., Stochastic dominance tests for decreasing absolute risk aversion II: General random variables, Management Science, 1977.

7. Yaari., M. E., Some remarks on measures of risk aversion and on their uses, Journal of Economic Theory, 1969.

8. www.bvb.ro 